\title{
Le Paysage Monte Alen/Monts De Cristal
}

\section{Chrislain Eric Kenfack}

\section{Relief et hydrographie}

Le paysage Monte Alén/Monts de Cristal (MA/MC) est à cheval sur le Sud-Est équato-guinéen et le Nord-Ouest gabonais. II couvre une superficie de $26747 \mathrm{~km}^{2}$, avec environ une moitié au Gabon et l'autre moitié en Guinée équatoriale. Il est formé de 3 écorégions, à savoir :

- L'écorégion des forêts congolaises atlantiques ;

- L'écorégion côtière équatoriale occidentale centrale ;

- L'écorégion côtière équatoriale occidentale méridionale.

Le paysage est situé sur une zone de plateaux et de chaînes montagneuses accidentées. Sur l'ensemble du paysage, la pluviométrie moyenne annuelle varie de $2000 \mathrm{~mm}$ à l'Est à 2 $800 \mathrm{~mm}$ à l'Ouest. La saison sèche va de juillet à septembre, cependant, elle est largement atténuée par la présence des nuages bas qui enveloppent une bonne partie du site. Les concessions forestières couvrent $65 \%$ du paysage, les aires protégées, $18 \%$, et seulement $3 \%$ des superficies sont dédiées à l'agriculture. Du côté gabonais, deux barrages hydroélectriques ont été construits dans la vallée de Mbé, pour alimenter la capitale Libreville (Etat des forêts 2006).

\section{Populations}

On retrouve deux grands groupes ethniques dans le paysage : les Fangs dans les zones montagneuses, et les Ndowe dans le bassin côtier en Guinée équatoriale. Les Beyele, minorité pygmée vivant jadis dans la région d'Altos de Nsork, ont migré vers le Sud-Cameroun, ou ailleurs dans la forêt depuis une vingtaine d'années.

Selon les dernières statistiques disponibles, datant de 2006, la densité moyenne de la population est de 16 à 18 habitants au $\mathrm{km}^{2}$ du côté équato-guinéen, et de 0,6 habitant au $\mathrm{km}^{2} \mathrm{du}$ côté gabonais.

\section{Couvert forestier}

Le couvert forestier du paysage Monte Alén/Monts de Cristal est de 26101 km² (Etat des forêts 2008). La végétation est assez diversifiée et riche. On y retrouve principalement :

- La forêt humide de basse terre,

- La forêt humide dégradée de basse terre,

- La forêt de montagne,

- La forêt dégradée de montagne,

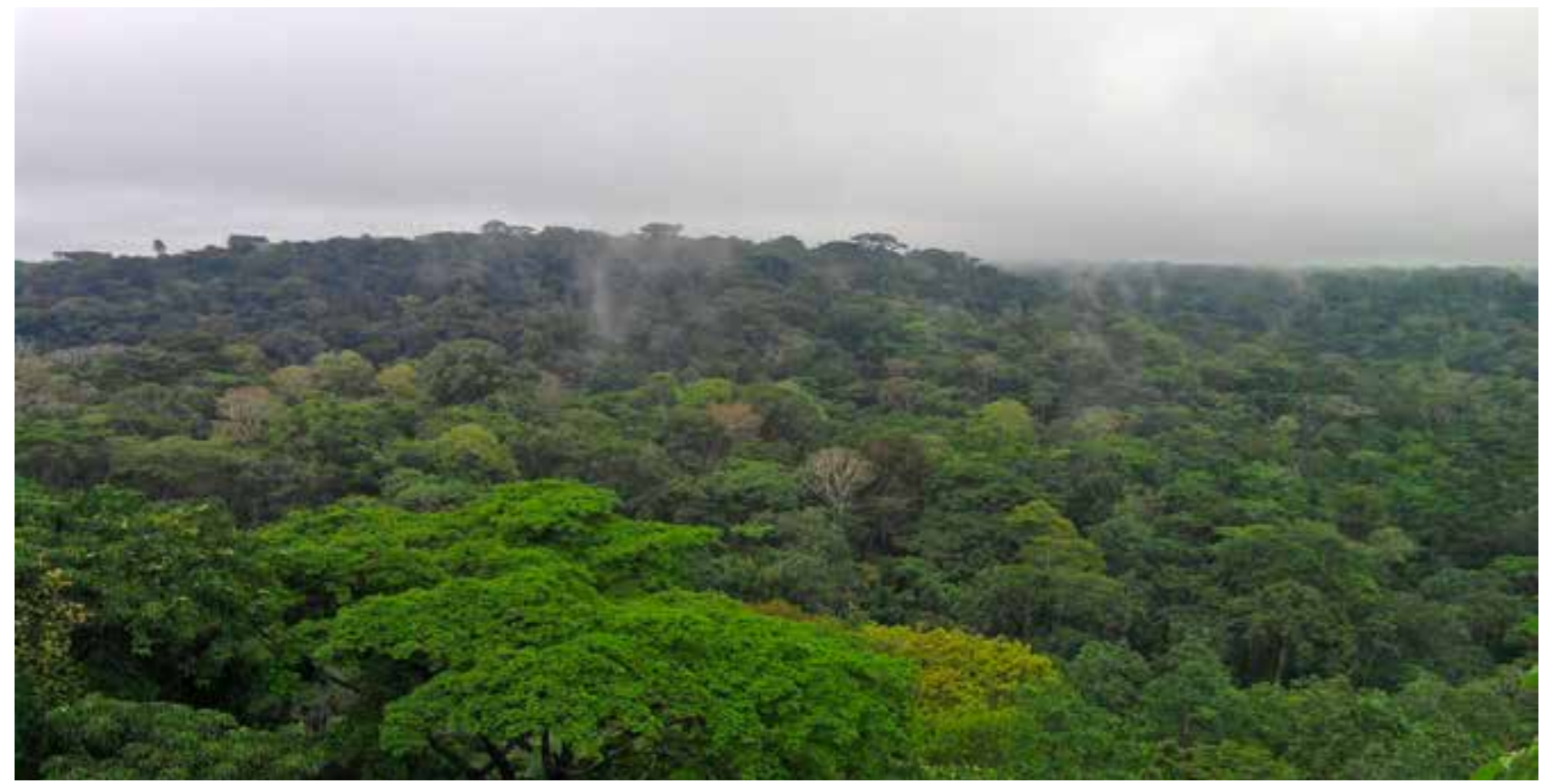


2

No. 19

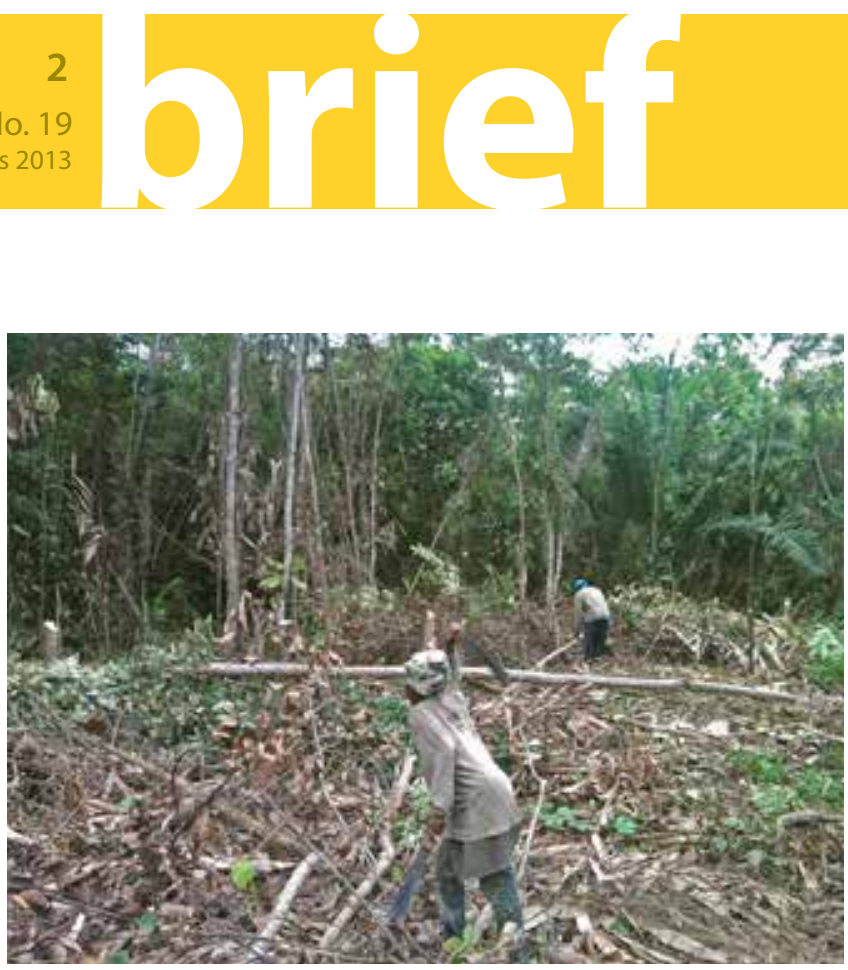

Préparation des champs pour l'agriculture familiale

- La forêt secondaire,

- La savane dans les prairies,

- Environ 500 ha d'Okoumé abandonnés.

\section{Déforestation}

La déforestation est l'un des principaux défis auxquels fait face le paysage. Entre 1990 et 2000, il a été enregistré une perte de $128 \mathrm{~km}^{2}$ de forêt, soit un taux de déforestation de 0,49\% (Etat des forêts 2008.

Les activités agricoles, principalement de subsistance, sont en partie responsables de cette déforestation. Cependant, celle-ci est due en grande partie à l'exploitation industrielle du bois par les sociétés forestières et à l'exploitation artisanale du bois pour des besoins énergétiques et artisanaux.

\section{Biodiversité}

Le paysage MA/MC est caractérisé par une biodiversité exceptionnellement riche. En effet, on y retrouve :

- $\quad$ Plus de 35 espèces de mammifères dont environ 10 espèces de primates, parmi lesquels les Gorilla gorilla, Pantroglodyte, Colobus satanas, Mandrillus sphinx, Cercocebus torquatus, Panthera pardus, Felis aurata, Loxodonta africana ;

- $\quad$ Plus de 246 espèces d'oiseaux, parmi lesquels les Phyloscopus budogoensis, Batis minima, Batis poensis, Malimbus racheliae;

- Plus de 25 espèces de reptiles ;

- Plus de 27 espèces d'amphibiens, parmi lesquels les Pedropedetes palmipes et les Leptodactylodon steverti;

- Une variété endémique de papillons, parmi lesquelles les Cymothoe haimodia (Nymphalidae), les Abantis eltringhami (Hesperidae) et les Saturnidae (Etat des forêts 2006).

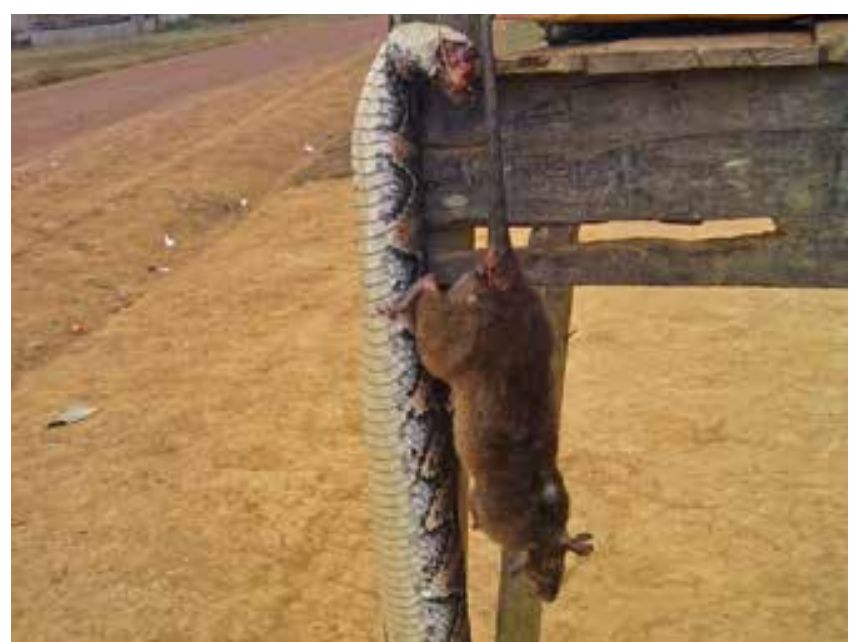

Viande de brousse en vente le long des villages

\section{Menaces pour la conservation de la biodiversité}

Les principales menaces qui guettent le paysage sont entreautres:

- La chasse illégale et le commerce de la viande de brousse, suite à l'augmentation du pouvoir d'achat des populations en Guinée équatoriale et la vente de l'ivoire dans la partie gabonaise du paysage ;

- L'exploitation industrielle et non durable du bois, du fait de la faible observance, par les exploitants forestiers, des lois et réglementations en matière de conservation ;

- Le manque de financement stable et de personnel qualifié et suffisant pour le contrôle du paysage ;

- L'expansion agricole ;

- L'exploitation minière artisanale et industrielle ;

- L'extraction non durable des PFNL pour des besoins de consommation et de commercialisation.

\section{Affectation et administration des terres}

On peut trouver les types d'affectation suivants:

3 parcs nationaux

- Le parc national de Monte Alén en Guinée équatoriale, d'une superficie de 200000 ha, qui constitue l'un des réservoirs les plus importants de biodiversité du Bassin du Congo, grâce à une très grande diversité floristique et d'espèces endémiques. Par ailleurs, il possède l'un des taux les plus élevés de rencontre des éléphants de forêt en Afrique.

- Le parc national d'Altos de Nsork en Guinée équatoriale, d'une superficie de 70000 ha, dont la végétation est une 
forêt humide d'altitude. Le parc abrite une population de gorilles des plaines actuellement menacée d'extinction, des animaux emblématiques tels que les chimpanzés, mandrills, colobus, léopards et éléphants.

- Le parc national des Monts de Cristal, d'une superficie de 120000 ha, se situant au Gabon (UNESCO, Convention du patrimoine mondial et COMIFAC 2008 ; Ko 2011). Des parcs de ce paysage, celui-ci est le plus inaccessible à I'homme, à cause de son bloc de forêts pluviales et de son relief allant de 200 à 900 mètres d'altitude, constamment couvert de nuages et de brume. Il est par conséquent un territoire presque vierge.

3 réserves

- La réserve militaire Monts de Cristal, dont la création se fait avec le soutien du WCS (Wildlife Conservation Society) dans la partie gabonaise.

- Le monument national Pidra Nzas en Guinée équatoriale, avec une superficie de 19000 ha.

- La réserve naturelle de Rio Muni en Guinée équatoriale, avec une superficie de 70000 ha (Etat des forêts 2006).

Outre ces parcs et réserves, le plan d'affectation des terres du paysage a délimité six macro- zones, réparties comme suit :

Trois zones de gestion communautaire des ressources naturelles (GCRN)

- La zone de Kougouleu-Medouneu-Mbe

- La zone du bosque nacional de Guinea equatorial

- La zone de la rivière Abanga

Trois zones d'extraction des ressources naturelles (ZER)

- La zone de Lonmin

- La zone de SEEF

- La zone de Rougier

\section{Partenaires institutionnels}

Les principaux partenaires institutionnels que l'on retrouve dans le paysage MA/MC sont entre autres:

- ECOFAC : Ecosystèmes fragilisés d'Afrique centrale

- WCS : Wildlife Conservation Society

- WWF : World Wide Fund for nature

- UICN/CARPE : Union internationale pour la conservation de la nature/ Central Africa Regional Program for the Environment (Programme régional de l'Afrique centrale pour l'environnement)

- MBG : Missouri Botanical Garden (jardin botanique de Missouri)

- INDEFOR : Instituto Nacional de Desarollo Forestal y Gestión del Sistema de Áreas Protegidas (Institut national pour le développement forestier et la gestion des aires protégées)

- IUBioma : Institut national de la biodiversité

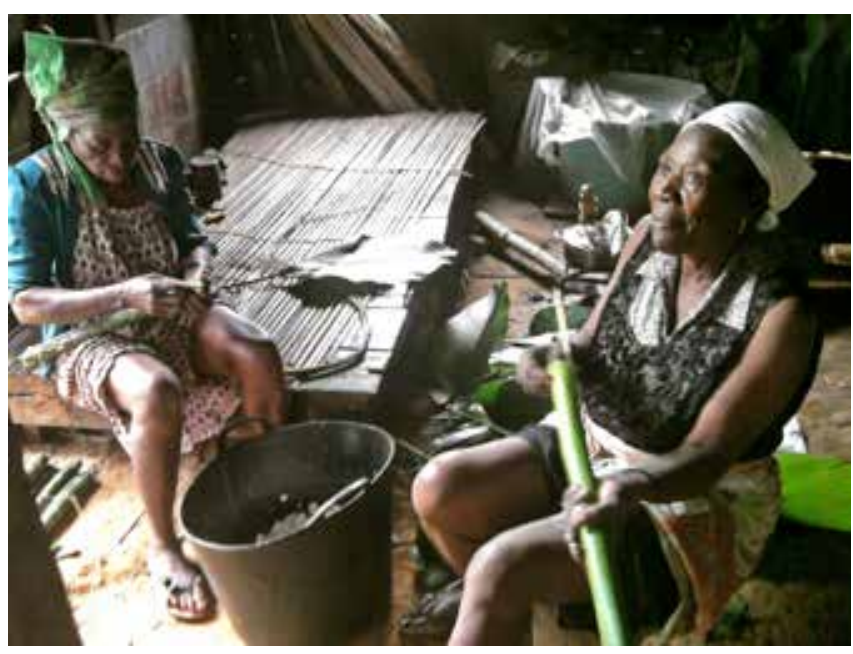

Préparation du manioc

\section{Opportunités, adaptation et REDD+}

Le paysage MA/MC présente certaines caractéristiques importantes à considérer pour la mise sur pied d'activités d'adaptation et de REDD+. Notamment :

- La faible densité de la population dans l'ensemble du paysage, comparativement aux autres paysages du Bassin du Congo. Cette faible densité de la population réduit la pression sur les forêts et sur les ressources naturelles et forestières du paysage, diminuant les fuites potentielles dans les projets REDD+.

- On compte, tant du côté gabonais qu'équato-guinéen, un ensemble de règles et de lois qui, bien appliquées, pourraient faciliter le déroulement des activités relatives à l'adaptation et à la REDD+.

- $\quad$ Il existe des plans d'aménagement et d'utilisation des terres dans toutes les aires protégées du paysage, qui pourraient entraîner une nette réduction de la déforestation s'ils étaient mis en application.

- L'exécution de projets visant à diversifier les options de subsistance telles que l'élevage de chèvres et de porcs, ainsi que l'horticulture dans certaines zones du paysage. Ces activités facilitent l'adaptation des populations face à la diminution et aux difficultés d'accès aux ressources naturelles et forestières du paysage.

\section{Défis et contraintes pour la REDD+ et l'adaptation}

Parmi les plus grands défis que rencontre le paysage MA/MC, on peut citer :

- La faible participation des populations aux activités de gestion et de conservation du paysage, surtout des aires protégées qui se trouvent à l'intérieur de celui-ci. II devient 
dès lors difficile de les impliquer dans les activités d'adaptation et d'atténuation axées sur la gestion des ressources naturelles situées dans la communauté.

- $\quad$ une multitude de partenaires et de projets qui opèrent dans l'individualisme. Le challenge serait de procéder à une coordination effective de l'ensemble des activités et des partenaires intervenant dans les zones, pour obtenir des résultats synergiques et une intervention homogène qui puisse améliorer les capacités d'adaptation.

- Les divergences sur le tracé des limites fiables entre les deux pays au niveau du paysage MA/MC. Or, ce tracé permettrait d'éviter les incompréhensions et l'amélioration de la gestion du paysage, de ses réserves et parcs.

- La non-maîtrise des lois et règlements en vigueur par les populations vivant dans et autour du paysage, ainsi que le non-respect de ces règles par les sociétés d'exploitation. Cette situation entraîne l'exploitation et I'utilisation abusives des ressources tant par les populations que par les sociétés d'exploitation opérant dans le paysage, réduisant le succès des interventions d'adaptation et d'atténuation.

\section{Documents consultés}

Accord sur la conservation des gorilles et de leurs habitats. Plan d'action 2008-Gorilles des plaines occidentales, Rome, 29 Novembre 2008, http://www.cms.int/species/gorillas/mop1/action_plan_ggg_ west_f.pdf, consulté le 20 Septembre 2012.

Denvers, D. et Vande Weghe, J. P. (coord.) 2007 Les forêts du Bassin du Congo : Etat des forêts 2006. Office des publications de I'Union européenne, Luxembourg.

De Wasseige, C. et Denvers, D. (éd.) 2009 Les forêts du Bassin du Congo : Etat des forêts 2008. Office des publications de I'Union européenne, Luxembourg.

De Wasseige, C. et Denvers, D. (éd.) 2011 Les forêts du Bassin du Congo : Etat des forêts 2010. Office des publications de I'Union européenne, Luxembourg.

Ko, J. 2011 Rapport annuel 2012: Activités USAID/ CARPE dans les paysages COMIFAC/ PFBC à l'appui du plan de convergence de la COMIFAC (septembre 2009- septembre 2010).

Les Monts de Cristal, une nature vierge et préservée, juin 2011, http:// www.parcsgabon.org/decouvrez-les-parcs/les-13-parcs-nationaux/ parc-national-des-monts-de-cristal, consulté le 20 septembre 2012.

White, L. et Vande Weghe, J .P. 2008 Patrimoine mondial naturel d'Afrique centrale : biens existants, biens potentiels, Rapport de l'atelier de Brazzaville du 12 au 14 mars 2008. Organisation des Nations Unies pour l'éducation, la science et la culture (UNESCO), Paris, Convention du patrimoine mondial et Commission des forêts d'Afrique centrale (COMIFAC), Yaoundé.
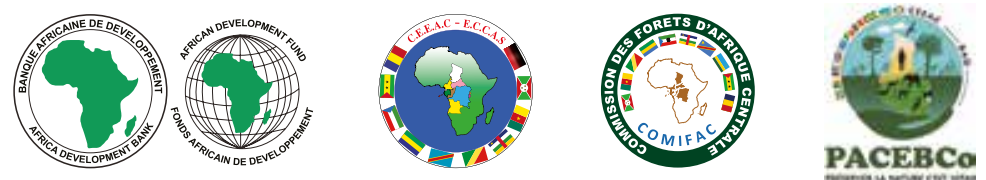

cifor.org/cobam 